\title{
NOUVELle
}

\section{Interleukine-4 et fusion des cellules musculaires}

Sophie B.P. Chargé
Molecular Medicine Program,

Ottawa Health Research

Institute, Ottawa, Ontario

KlH 8L6, Canada.

scharge@ohri.ca
> La fusion des cellules musculaires survient durant le développement des muscles squelettiques et, chez l'adulte, lors de l'adaptation du muscle adulte à un traumatisme musculaire ou à un exercice physique intense. Au cours de ces différents processus, les cellules myogéniques mononucléées (myoblastes ou cellules satellites) sont activées, prolifèrent, se différencient et fusionnent, entraînant la formation et l'hypertrophie des cellules musculaires plurinucléées (myofibres) (Figure 1). Les myofibres sont des compo- santes essentielles des muscles squelettiques qui permettent la contraction musculaire. Par conséquent, la régulation de leur taille est nécessaire au bon fonctionnement du muscle squelettique. À ce jour, plusieurs facteurs de croissance, dont I'IGF-1 (insulin-like growth factor-1), et I'HGF (hepatocyte growth factor), ont été impliqués dans le processus d'activation des cellules myogéniques. Toutefois, la fusion des cellules myogéniques est un processus plus complexe qui requiert l'orchestration d'une série d'événements cel-

laires qui contrôlent ces processus de fusion myogénique sont peu connus. Cependant, une partie de ce puzzle vient d'être découverte. $\varepsilon$ n effet, une récente publication implique la voie de signalisation de NFATc2 (nuclear factor of activated $T$ cells) dans l'activation du gène de l'interleukine-4 (IL-4) et la fusion des cellules musculaires.

Cette voie de signalisation fait intervenir la calcineurine, une phosphatase de type sérine/thréonine, qui est un médiateur essentiel de la signalisation calcique. Ainsi, l'activation de la calcineurine permet la déphosphorylation des facteurs de transcription NFAT ; cela démasque le signal NLS (signal de localisation nucléaire) et permet l'association de ces facteurs NFAT avec l'importine et leur transport dans le noyau où ils activent alors la transcription de leurs gènes cibles. La famille des facteurs de transcription NFAT comprend plusieurs isoformes (NFATcl-c4) ayant chacune un rôle cellulaire propre. Ainsi, des études in vitro ont montré la translocation spécifique de certaines isoformes vers le noyau au cours des différents stades de la différenciation myogénique en réponse au signal calcique [1]. NFATcl et NFATc2, exclusivement 
cytoplasmiques au stade de myoblastes, sont transportées dans les noyaux des myotubes nouvellement formés lors de la différenciation myogénique. À l'inverse, NFATc3 est nucléaire dans les myoblastes et cytoplasmique après fusion. Ces résultats suggèrent fortement que ces facteurs de transcription ont des rôles distincts durant la différenciation des cellules myogéniques.

D'autres études in vivo et in vitro ont montré l'importance des facteurs NFAT dans la régulation de l'hypertrophie musculaire et la spécification des fibres musculaires en type lent ou rapide (voir Encadré). Par exemple, l'inhibition de la calcineurine par injection de ciclosporine $A(C s A)$ provoque une inhibition de I'hypertrophie musculaire et une transformation des fibres musculaires de type lent en un type rapide (pour revue, voir [2]). Cependant, le rôle de l'activation des NFAT dans cette adaptation physiologique in vivo reste à démontrer. Plus importante est l'observation qu'une stimulation électrique des fibres musculaires en culture, qui mime un signal de type lent, induit la translocation rapide de NFATcl vers le noyau. Dans ce modèle, la translocation de NFATcl est sensible à la CsA, confirmant le rôle de la calcineurine dans l'activation de NFATcl [3]. Ces résultats suggèrent donc un rôle pour NFATcl dans la spécification des fibres lentes. En revanche, les souris mutantes qui n'expriment pas les gènes NFATC2 ou NFATC3 ont une répartition normale des fibres musculaires de type lent et rapide, mais présentent une réduction de la masse musculaire secondaire à une diminution de la taille des fibres musculaires (mutant NFATC2) et du nombre total de fibres musculaires (mutant NFATC3) [4, 5]. Ces études sont en accord avec la localisation des isoformes in vitro.

Si ces résultats confirmaient le rôle spécifique des différentes isoformes NFAT dans la régulation de la myogenèse, il restait à en identifier les gènes cibles. Récemment, V. Horsley et al. ont démontré que le gène codant pour l'IL-4 est sous le contrôle de NFATc2 dans les cellules musculaires [6]. En effet, contrairement aux myotubes issus de souris sauvages, ceux de souris NFATC2 ${ }^{-/-}$n'expriment pas l'IL-4, et l'expression de I'ADNc NFATC2 dans ces mêmes cellules active l'expression de I'IL-4 endogène. Cette étude est la première démonstration de l'expression d'IL-4 par une cellule qui n'appartient pas au système immunitaire et suggère donc un nouveau rôle pour cette molécule. La taille des myotubes NFATC2 ${ }^{-1-}$ est inférieure à la normale, mais elle est corrigée par l'ajout de doses physiologiques d'IL-4 au milieu de culture. De plus, en immunomarquage, I'IL-4 est détectée presque exclusivement dans les myotubes nouvellements formés. Enfin, la preuve de l'action spécifique de l'IL-4 sur les cellules myogéniques mononucléées a été apportée dans des expériences de coculture de myotubes normaux avec des myoblastes $I L-4 R \alpha^{-/-}$. On observe une réduction très importante de la fusion des myoblastes $I L-4 R \alpha^{-/-}$avec les myotubes. À l'inverse, la fusion des myoblastes normaux aux myotubes $I L-4 R \alpha^{-/}$reste inchangée. L'IL-4 a un rôle similaire in vivo. On constate en effet dans les muscles des souris mutantes pour les gènes $I L-4$ et $I L$ $4 R \alpha$ une réduction de la taille des fibres musculaires due à une réduction de la fusion myogénique. En conclusion, les auteurs montrent que I'IL-4, sécrétée par les cellules musculaires nouvellement formées (myotubes), interagit avec les récepteurs de cette cytokine (IL-4R $\alpha$ ) exprimés sur les myoblastes, et promeut la fusion de ces derniers avec les myotubes (Figure 1). Ce processus permet ainsi la croissance des fibres musculaires et donc l'augmentation de la masse musculaire.

On peut donc faire un parallèle entre le processus de fusion des myoblastes et celui des macrophages, ces deux types de cellules partageant une voie de signalisation commune. En effet, l'IL-4, initialement identifiée dans les lymphocytes Th2 ( $T$ helper), est impliquée dans l'activation des macrophages durant la réponse immune et, avec I'IL-13, favoriserait la fusion des macrophages. II n'est donc pas exclu que, comme cela vient d'être démontré dans le cas du foie $(\rightarrow)$, la participation des cellules hématopoiétiques de la moelle osseuse à la régénération du muscle $(\rightarrow) \mathrm{m} / \mathrm{s}$ 2003, n० 6-7, p. 683 soit la conséquence d'une fusion illégitime des macrophages avec les myotubes et non d'une différenciation myogénique de ces cellules souches [7]. Cette hypothèse n'est cependant pas confirmée par les résultats d'une étude dont l'objectif était d'analyser spécifiquement in vivo ce processus de fusion (voir à ce propos la Brève, p. 1201 de ce numéro) [8]. Mais si la fusion des myoblastes/myotubes est contrôlée par l'IL-4, tel n'est pas le cas de la fusion myoblaste/myoblaste, ce qui suggère l'existence de deux voies de signalisation distinctes contrôlant le processus de fusion dans le muscle de la souris. Cette observation est en accord avec l'étude faite récemment démontrant le rôle de la molécule Rolling pebbles dans la fusion myoblaste/myotube chez la drosophile [9].

Ces nouveaux résultats suggèrent aussi que les cytokines sécrétées au cours de la réponse immunitaire et/ou inflammatoire accompagnant un traumatisme musculaire, jouent un rôle plus important qu'il n'avait été précédemment reconnu dans la régulation de la régénération musculaire. En effet, des études antérieures avaient montré le rôle des effecteurs de la réponse immune dans la prolifération des myoblastes [10], ce que confirment $V$. Horsley et al. pour I'IL-4 [6]. Les cytokines auraient donc une double fonction, synchronisant les différentes phases de prolifération et de fusion observées durant la régénération musculaire.

L'identification d'un des gènes cibles de NFATc2 ouvre la voie à l'identification prochaine des autres intermédiaires contrôlant la fusion entre les myoblastes et les myotubes. On sait déjà que les kinases Janus, l'insulin receptor sub- 
strate-1/2, la PI(3) kinase, la MAP kinase et Stat-6 sont des gènes cibles en aval de la voie de signalisation activée par I'IL-4R dans d'autres cellules [11]. En est-il de même durant la fusion myoblaste/myotube? L'activation de la voie de signalisation $\mathrm{PI}(3)$ kinase est particulièrement intéressante car elle est impliquée dans l'hypertrophie musculaire en réponse au signal d'IGF-1 (insulin growth factor-1). Un intermédiaire supplémentaire est représenté par les intégrines, responsables des interactions cellule/cellule et matrice extracellulaire/cellule et qui facilitent la fusion cellulaire. Les intégrines $\beta 1$ et $\beta 2$ sont également activées par l'IL-4 lors de la fusion des macrophages [12]. En outre, le signal déclenché lors de l'activation des intégrines est impliqué dans le développement des muscles squelettiques et une étude récente a montré l'importance de l'intégrine $\beta 1$ dans la fusion des myoblastes [13]. Il est donc problable que, comme dans les macrophages, I'IL-4 contrôle l'expression du gène codant pour l'intégrine $\beta \mathrm{l}$ durant la fusion des myoblastes.

Parmi les perspectives thérapeutiques qu'offrent ces travaux, on peut imaginer que la stimulation de la voie de signalisation en aval de I'IL-4R $\alpha$ permettrait de stimuler la croissance du muscle squelettique. Ceci permettrait de prévenir la perte de masse musculaire observée dans certaines myopathies ou au cours du viellissement. Plus encore, le développement de stratégies d'augmentation de la fusion des myoblastes permettrait peut-être d'améliorer l'implantation de myoblastes ou de cellules souches dans les muscles des patients atteints de myopathies. $\diamond$

Unexpected function for IL-4

in muscle cell fusion

- Les fibres de typel ou fibres lentes sont très vascularisées et riches en mitochondries, permettant l'utilisation de l'oxygène comme source d'énergie pour produire leurs contractions. Elles sont donc peu fatigables. De plus, l'expression de la myosine de type I leur confère des caractéristiques de fibres à contractions lentes. Elles sont donc adaptées aux efforts soutenus (épreuves d'endurance) et au maintien de la posture.

- Les fibres de typell ou fibres rapides sont fortement pourvues en enzymes permettant I'utilisation du glycogène comme source d'énergie en l'absence d'oxygène. Elles sont donc plus rapidement fatigables. Cependant, l'expression de la myosine de type Ila, Ilb ou IIx leur permet une contraction rapide adaptée aux activités physiques intenses mais de plus courte durée.

\section{RÉFÉRENCES}

1. Abbott KL, Friday BB, Thaloor D, Murphy TJ, Pavlath GK. Activation and cellular localization of the cyclosporine A-sensitive transcription factor NF-AT in skeletal muscle cells. Mol Biol Cell $1998 ; 9$ : 2905-16.

2. Schiaffino $S$, Serrano A. Calcineurin signaling and neural control of skeletal muscle fiber type and size. Trends Pharmacol Sci 2002; $23: 569-75$.

3. Liu Y, Cseresnyes Z, Randall WR, Schneider MF. Activitydependent nuclear translocation and intranuclear distribution of NFATc in adult skeletal muscle fibers. J Cell Biol $2001 ; 155: 27-39$.

4. Horsley V, Friday BB, Matteson S, Kegley KM, Gephart J, Pavlath GK. Regulation of the growth of multinucleated muscle cells by an NFATC2-dependent pathway. J Cell Biol 2001 ; 153: 329-38.

5. Kegley KM, Gephart J, Warren GL, Pavlath GK. Altered primary myogenesis in $\operatorname{NFATC3}(-/-)$ mice leads to decreased muscle size in the adult. Dev Biol $2001 ; 232$ : 115-26.
6. Horsley V, Jansen KM, Mills ST, Pavlath GK. IL-4 acts as a myoblast recruitment factor during mammalian muscle growth. Cell 2003 ; $113:$ 483-94.

7. LaBarge MA, Blau HM. Biological progression from adult bone marrow to mononucleate muscle stem cell to multinucleate muscle fiber in response to injury. Cell 2002; 111 : 589-601.

8. Alvarez-Dolado M, Pardal R, Garcia-Verdugo JM, et al. Fusion of bone-marrowderived cells with Purkinje neurons, cardiomyocytes and hepatocytes. Nature 2003; 425: 968-73.

9. Rau A, Buttgereit D, Holz A, et al. Rolling pebbles (rols) is required in Drosophila muscle precursors for recruitment of myoblasts for fusion. Development 2001 ; 128 : 5061-73.

10. Hawke TJ, Garry DJ. Myogenic satellite cells: physiology to molecular biology. J Appl Physiol 2001 ; 91 : 534-51.

11. Nelms K, Keegan AD,
Zamorano J, Ryan JJ, Paul WE. The IL-4 receptor: signaling mechanisms and biologic functions. Annu Rev Immunol 1999; 17 : 701-38.

12. McNally AK, Anderson JM. Betal and beta2 integrins mediate adhesion during macrophage fusion and multinucleated foreign body giant cell formation. Am J Pathol $2002 ; 160: 621-30$.

13. Schwander M, Leu M, Stumm $M$, et al. Betal integrins regulate myoblast fusion and sarcomere assembly. Dev Cell $2003 ; 4: 673-85$.

\section{REMERCIEMENTS}

Je remercie Guillaume Grenier et Muriel Amar pour leurs corrections. 